\title{
A RAT MODEL OF HUMAN FENIB (familial encephalopathy with neuroserpin inclusion bodies)
}

Katsura Takano ${ }^{\#+}$, Yasuko Kitao ${ }^{\#+}$, Reiko Inagi $i^{\sharp}$, Takashi Momoi ${ }^{\&}$, Tomohiro Matsuyama $^{\%}$, Toshio Miyata ${ }^{\ddagger}$, Yukio Yoneda ${ }^{\$}$, Hiroyuki Iso ${ }^{+}$, David M. Stern ${ }^{=}$, Osamu Hori", and Satoshi Ogawa ${ }^{\#}$

\footnotetext{
\#Department of Neuro anatomy, Kanazawa University Med ical School, Ishikawa, Japan

${ }^{\circledR}$ The $21^{\text {st }}$ Century COE Program on Innovative Brain Sciences for Development, Learning, and Memory, Japan

\$Laboratory of Molecular Pharmacology, Kanazawa University Graduate School of Natural Science and Technology, Ishikawa, Japan,

${ }^{+}$Department of Behavior Science

${ }^{\%}$ Department of Medicine, Hyogo Medical College, Hyogo, Japan,

${ }^{\&}$ Division of Development, National Institute of Neuroscience, Tokyo, Japan.

${ }^{*}$ Molecular and Cellular Nephrology, Institute of Medical Sciences, Tokai

University, Kanagawa, Japan, and

=Dean's Office, University of Cincinnati College of Medicine, OH, USA

${ }^{+}$both authors equally contributed to this work.
}

Running title: ER stress and serpinopathy

Please address correspondence to:

Yasuko Kitao Ph.D.

Department of Neuroanatomy, Kanazawa University Medical School, 13-1

Takaramachi, Kanazawa City, 920-8640, Japan.

Tel: +81-76-265-2162 Fax: +81-76-234-4222

e-mail: kitao@nanat.m.kanazawa-u.ac.jp

Abbreviations: ER, endoplasmic reticulum; GRP, glucose-regulated protein; ORP, oxygen-regulated protein; SNpc, substantia nigra pars compacta; Tg, transgenic; TH, tyrosine hydro xylase. 


\begin{abstract}
FENIB (familial encephalopathy with neuros erpin inclusion bodies) is caused by intracellular accumulation/polymerization of mutant neuroserpin s in the endoplasmic reticulum (ER). Transgenic rats overexpress ing megsin (Tg meg), a newly identified serine protease inhibitor (serpin), demonstrated intraneuronal periodic-acid Schiff (PAS)-positive inclusions distributed throughout deeper layers of cerebral cortex, CA1 of the hippocampus, and substantia nigra. Hippocampal extracts from Tg meg rats showed increased expression of ER stress proteins, and activation of caspases-12 \& -3 , associated with decreased neuronal density. Enhanced ER stress was also observed in dopaminergic neurons in the substantia nigra, in parallel with decreased neuronal viability and motor coordination. In each case, PAS-positive inclusions were also positive for megsin. These data suggest that overexpression of megsin results in ER stress, eventuating in the formation of PAS-positive inclusions. Tg meg rats provide a novel model of FENIB, where accumulation of serpins in the ER induces selective dysfunction/loss of specific neuronal populations.
\end{abstract}

Key words: neuroserpin, protein malfolding, neuronal cell death, oxygen/glucose regulated protein (ORP/GRP), oxidant stress.. 


\section{INTRODUCTION}

Serpinopathy is a newly identified class of disorders characterized by abnormal accumulation of serine protease inhibitors or serpins [1]. Serpins comprise a broad superfamily of proteins found in a range of species, and include $\alpha_{1}$-antitryps in, C1 esterase inhibitor, antithrombin III and plasminogen activator inhibitor-1. Consistent with their diverse functions, mutations in alleles encoding serpins have systemic effects, as demon strated by the clinical syndrome accompanying $\alpha_{1}$-antitrypsin deficiency [2]. Mutant serpins have a tendency to aggregate, forming intracellular inclusions in the endoplasmic reticulum (ER), and are associated with cellular degeneration. For example, Collins bodies, due to aggregation of neuroserpin (a brain-specific serpin synthesized and secreted by neurons), are closely associated with mutations homologous to those which cause aggregation of $\alpha_{1}$-antitrypsin in hepatocyes [3, 4]. The accumulation of neuroserpin results in formation of PAS-positive inclusions in the brain and is associated with a neurodegenerative disorder termed familial encephalopathy with neuroserpin inclusion bodies (FENIB) [5].

We have previously cloned from cultured human mesangial cells and characterized a novel member of the serpin superfamily, megsin $[6,7]$. To better define its characteristics, a rat model was developed with genetic overexpression of megsin in multiple cell types (Tg meg rats). Quite unexpectedly, this experimental model has characteristics of a serpinopathy [8]. In homozygous Tg meg rats, PAS-positive, diastas e-resistant intracellular droplets developed in the kidneys and pancreas, and were accompanied by marked up-regulation of ER stress chaperones, suggesting the association of ER stress with this new type of serpinopathy [9]. 
While homozygous $\mathrm{Tg}$ meg rats displayed early and progressive organ damage, hetrozygotes failed to show abnormalities in either renal or endocrine function.

However, although heterozygotes had a similar life span to nontransgenic (nonTg) littermates, they demonstrated PAS-positive intracellular inclusion in neurons and slowly progressive neurodegeneration, accompanied by upregulation of an ER resident stress protein, ORP150/HSP12A (150 kDa oxygen-regulated protein) [10], and activation of caspase-12, a cell death enzyme activated by ER dysfunction [11]. These findings lead us to propose that ER stress may have a pathogenic role in the development of serpinopathy, ultimately leading to neurodegeneration [4]. 


\section{MATERIALS AND METHODS}

Megsin transgenic rats (Tg meg) were established as described [8]. Offspring that carried the transgene were identified by Southern blotting of genomic DNA extracted from tails, as described, or Western blot analysis [7]. Animals were monitored for pancreatic and renal function by measuring blood urea nitrogen and glucose levels [8] up to 12 months after birth. Animals with signs of either pancreatic or renal dysfunction were excluded from this study. Heterozygotes were maintained by crossing animals with wild-type Wistar rats. Homozygous Tg meg rats failed to survive up to reproductive age.

Western blotting. Levels of ER stress proteins in cell or tissue extracts were determined by immunoblotting, as described [10], using either anti-human ORP150 antibody $(1 \mu \mathrm{g} / \mathrm{ml})$ or anti-KDEL monoclonal antibody (Stress gen Biotecnologies Co., Canada ; $0.2 \mu \mathrm{g} / \mathrm{ml})$, the latter to assess levels of GRP78 [12]. Immunoblotting also employed either anti-activated caspase- 12 antibody [11], anti-activated caspase-3 antibody [13], or anti-CHOP antibody [14]. Blots were reacted with antibody to $\beta$-actin as an internal control (1000x dilution, Sigma, St. Louis., MI).

Behavioral analysis. Motor coordinate ability was determined using the pole test, as described [15]. In brief, animals were placed head upward near the top of a rough-surfaced wood pole $(5 \mathrm{~cm}$ in diameter and $100 \mathrm{~cm}$ in height), and the time required for animals to turn completely downward and climb down the floor was recorded. Triplicate pole tests, with a four hour inter-trial interval, were carried out and average values were calculated.

Histochemistry and evaluation of cell death in vivo. Animals were perfused under deep anesthesia with chloral hydrate ( $400 \mathrm{mg} / \mathrm{kg}$ body weight, i.p.) through the 
ascending aorta with saline, followed by fixative ( $4 \%$ paraformaldehyde in $0.1 \mathrm{M}$ phosphate-buffered saline [PBS; pH 7.4]). Brains were removed, and after postfixation overnight at $4{ }^{\circ} \mathrm{C}$ and cryoprotection in $30 \%$ sucrose in PBS, serial coronal sections $(14 \mu \mathrm{m})$ were cut on a cryostat and mounted in a series of ten silanized slides. This approach produced ten sets of $140 \mu \mathrm{m}$ interval serial sections covering the whole extent of any brain nuclei. Each set of slide-mounted sections underwent histological processing for either PAS staining, cresyl violet staining or immunohistochemistry. Where indicated, sections were treated with diastase $(0.1 \%$, Wako, Tokyo, Japan $)$ at $37^{\circ} \mathrm{C}$ for 1 hour followed by the PAS staining [8]. Immunohistochemical analysis was performed as described [16]. In brief, sections were preincubated with $1 \%$ bovine serum albumin and $0.1 \%$ Triton X-100 in PBS, and then incubated with primary antibodies overnight at $4^{\circ} \mathrm{C}$. After washing, sections were treated with the appropriate Cy3 or FITC-conjugated secondary antibody (Jackson Immunoresearch, West Grove, PA) for 1 hour at room temperature, or with EnVision Kit (DAKO) for diaminobenzidine staining. To evaluate neuronal death in the hippocampus, sections were immunostained with anti-activated caspase-3 antibody [13]. Nissl-positive neurons were counted in hippocampal lesions, CA1 ( 0.5 and 2.0 from midline) and CA2/3 in slices obtained at the depth of $-3.30 \mathrm{~mm}$ from the Bregma, using a $0.4 \mathrm{~mm}$ span of the microscopic scale located parallel to the pyramidal layer. Sections were also double-stained with anti-Neu-N antibody (1:100 dilution; Chemicon, Temecula, CA, USA) [17] and anti-activ ated caspase-12 antibody. Neuronal death was assessed based on an overlapping distribution of activated caspase-12-positive and Neu-N-positive cells. Cell death in neurons of the substantia nigra pars compacta (SNpc) was evaluated by double-staining with anti-tyrosine hydroxylase (TH) antibody 
(Sigma-Aldrich, Inc., St. Louis, Mis souri, USA; 1:1,000 dilution) [18] and anti-activated caspase- 12 antibody. TH-positive neurons were counted in each coronal slice obtained at four different depths $(-4.8,-5.2,-5.6,-6.0) \mathrm{mm}$ from the Bregma) using Photoshop software (Adobe, Tokyo, Japan). The percent volume of TH-positive area was compared with control animals. In each case, two observers without knowledge of the experimental protocol evaluated sections and experiments were repeated at least three times.

In vitro studies. Astrocytes were prepared from cerebral cortices of rodents as described previously [10]. When astrocyte cultures, prepared from rats of the indicated genotype, achieved confluence, they were exposed to hypoxia using an incubator attached to a hypoxia chamber (Coy Laboratory Products, Ann Arbor MI), which maintained a humidified atmosphere with low oxygen tension (8-10 Torr) for up to $48 \mathrm{hrs}$ [21]. Cell viability at the indicated time point was ass essed by dye exclusion in astrocytes. Levels of ER stress proteins in astrocytes were determined by immunoblotting, as described above. Subcellular fractionation of astrocytes was performed using to OptiPrep (Life Technologies), as described [21]. In brief, astrocytes $\left(\sim 5 \times 10^{6}\right.$ cells $)$ were either exposed to hypoxia or maintained in normoxia for 24 hours, and homogenates were subjected to subcellular fractionation using a self-generated gradient. Fractions were then subjected to dot blot analysis using anti-megsin antibody. Samples were also used for dot blot analysis with anti-mouse calnexin antibody (Transduction Laboratories) and anti-mouse TGN38 antibody [23] (Transduction Laboratories) as markers for endoplasmic reticulum and Golgi apparatus, respectively. 
Statistical analysis was performed, unless indicated otherwise, by two-factor ANOVA followed by Scheffe's F test as post-hoc analysis. Where indicated, analysis was also performed by repeated measure ANOVA. 


\section{RESULTS}

Expression of megsin in the central nervous system is associated with the presence of PAS-positive inclusion bodies. Previously, we reported that homozygous Tg meg rats displayed severe pancreatic and renal dysfunction [8]. In $\sim 80 \%$ of hetero zygous Tg meg rats, blood glucose and blood urea nitrogen levels remain within the normal range for the first 12 months of life (not shown). Whereas non Tg littermates displayed virtually und etectable levels of megsin in the brain, consistent with our previous report [8], Tg meg heterozygotes showed, at 4 months of age, the expression of antigen throughout the brain, including cerebral cortex, cerebellum, midbrain, and hippocampus (Fig. 1A), and was accompanied by marked upregulation of an ER-stress marker, ORP150 [10]. Though no major brain developmental abnormalities were observed in hetero zygous Tg meg rats, PAS-positive inclusions were observed in specific subgroups of neurons, including CA1 of the hippocampus, d eeper layers (III - V) of the cerebral cortex, and the substantia nigra (Fig. 1B-D). No such inclusions were observed in either the cerebellar cortex, red nucleus, or brainstem reticular formation. Inclusions were not observed in white matter (topologic distribution is shown in Fig 1F). PAS-positive inclusions in CA1 (Fig. 1G, indicated by open arrowheads) were further analyzed immunohistochemically. ORP150 was markedly upregulated in CA1 (Fig. 1H) compared with nonTg controls (not shown). PAS-positive inclusions were also positive for megsin (Fig. 1I), and ORP150 was detected in these neurons, though the intensity of staining was somewhat less (Fig. 1J). PAS signals in Tg meg rats were resistant to diastase treatment (not shown) and no such inclusions were detected in wild-type littermates (Fig. 1K). 
Accelerated death of hippocampal neurons in Tg meg rats. Consistent with the evidence that presence of PAS-positive intracellular inclusions in FENIB is associated with neuronal degeneration [5], apparent neuronal loss was observed in the hippocampus CA1 region of 4-12 month old Tg meg rats, when compared with nonTg littermates (Fig. 2A-F, G). Immunoblotting revealed enhanced expression of GRP78 and ORP150 in the hippocampus, both of which are associated with stress responses originating from the ER $[19,20]$ (Fig. 3A \& B). ORP150 expression peaked at $\sim 2-4$ months after birth, while GRP78 levels remained elevated at all times in Tg meg rats, compared with nonTg con trols (Fig. 3A). In the cerebellar cortex, levels of ORP150 were somewhat higher in Tg meg animals, versus controls, though GRP78 expression was similar in both groups (Fig. 3B). In the hippocampus of Tg meg rats, activated caspase-12 antigen was detected at 2-6 months of age; i.e., after in itiation of ER stress, based on ORP150 and GRP78 levels (Fig. 3A). Activation of caspase-3 was also detected 2-6 months after birth. No immunoreactive activated caspase-12 was detected in the hippocampus of nonTg littermates (Fig. 3A). Furthermore, ER stress appeared to be concentrated in the hippocampus, as neither cerebellar cortex of Tg meg rats nor non Tg littermates displayed activated caspase-12 antigen (Fig. 3B). Similarly, no peak of ORP150 or activated caspase-12 antigen was seen in cerebellar cortex of either $\mathrm{Tg}$ meg or non Tg controls (Fig. 3B).

Based on these observations, it seemed likely that neurons might express markers of ER stress, as well as activated caspase-12 antigen, in hippocampii of Tg meg rats. Immunohistochemical analys is of 4 month old $\mathrm{Tg}$ meg rats demonstrated high levels of ORP150 in CA1 of the hippocampus (Fig. 3E). Immunostaining revealed increased activated caspase-3 antigen (Fig. 3F). Furthermore, double-stain ing with 
anti-Neu-N antibody (which specifically recognizes neuronal cell bodies) and anti-activated caspase- 12 antibody revealed a partially overlapping distribution of these signals (Fig. 3G-I). Increase of activated caspase-12 immunoreactivity in the CA1 area of $\mathrm{Tg}$ meg rats appeared to peak at 4 months after birth (Fig. 3C). These data suggested the possibility that an ER-mediated cell death pathway was operative in neuronal cells in Tg meg rats.

Neuronal cell death in the SNpc of Tg meg rats. Evaluation of other brain subregions demonstrated a distribution of PAS-positive inclusions in another population of neurons in the midbrain, especially in the substantia nigra pars compacta (SNpc; Fig. 1D, Fig. 4A). Four months after birth, inclusions were observed in neurons in SNpc (indicated by arrow), surrounded by normal neurons (indicated by arrowhead). No such inclusions were observed in nonTg animals (Fig. 4B). Immunohis tochemical analysis was performed with either anti-megsin antibody or antibody against tyrosine hydroxylase $(\mathrm{TH})$ [18], a marker of dopaminergic neurons. Consistent with our observations in CA1 (see above), intracellular inclusions were strongly positive for megsin (indicated by arrows in Fig. 4A, C \& E). In contrast, neurons which were negative for PAS-positive inclusions could be divided in two categories; i) those positive for megsin and TH (indicated by diamonds in Fig. 4A, C, D, \& E), and, ii) another population displaying weaker staining for megsin and high intensity staining for TH (indicated by arrowheads in Fig. 4A, D, \& E). These three populations were simultaneously distributed in the SNpc of 4 month old Tg meg rats, suggesting that chronic neurodegeneration might resu lt from repetitions of acute episodes of cell stress and neuronal death. Neuronal cell death was confirmed by TH immunostaining at 
variable periods after birth in Tg meg rats. TH immunostaining in Tg meg rats revealed a distribution of TH-positive neurons comparable to nonTg littermates at 2 months after birth. However, there was a dramatic decrease in TH-positive neurons at 6 months after birth (Fig. 4F-M). Semi-quantitative analysis showed dramatic loss of SNpc volume; $98.2 \pm 3.4,51.4 \pm 6.7,28.2 \pm 4.5,20.2 \pm 3.8(\mathrm{n}=6$, mean + S.D. $)$, at 2, 4, 8, and 12 months after birth, respectively. Statistical analys is indicates significant volume loss at 4,6 , and 12 months after birth ( $\mathrm{p}<0.01$ by Sheffe's analysis followed by ANOVA). The loss of TH immunointensity was accompanied by loss of motor coordination in $\mathrm{Tg}$ meg rats (Fig. $4 \mathrm{U} \& \mathrm{~V}$ ).

To further analyze neuronal death in the SNpc, we performed immunostaining with anti-ORP150 antibody, which showed marked upregulation at 4 months after birth, compared with nonTg littermates (Fig. 5B \& F). Immunostaining with anti-TH antibody demonstrated a decrease in TH-positive neurons in the SNpc (Fig. 5C \& G), accompanied by a partially overlapping pattern with activated caspase- 12 immunoreactivity (Fig. 5D). Quantitative analysis revealed activation of caspase-12 in TH-positive neurons reaching maximal levels by 4 months after birth in Tg meg rats (Fig. 5I). Thus, TH-positive neurons display activated caspase-12 antigen, and appear to die over time in Tg meg rats, correlating with loss of SNpc function.

Accumulation of megsin protein by ER-stress. Our findings, thus far, suggested the possibility that in Tg meg rats neuronal degeneration occurs (at an accelerated pace compared with nonTg controls) in the hippocampus and SNpc, and that this is preceded by the presence of megsin-containing inclusion bodies. To gain further insight into the relationship between overexpression of megsin and ER dysfunction, we utilized 
astrocytes (because of their ease of cultivation) from $\mathrm{Tg}$ meg rats. When cultured astrocytes from $\mathrm{Tg}$ meg rats were exposed to hypoxia, a stimulus associated with ER stress [24], cell survival was diminished compared with cultures from nonTg controls (Fig. 6A). Expression of ORP150 in cultured as trocytes from Tg meg animals was slightly higher under normoxic conditions than in nonTg controls, and reached peak levels earlier than controls on exposure to hypoxia. A similar pattern was observed with respect to expression of GRP78 in astrocytes. Mediators related to ER-dependent cell death, such as activated caspase-12 and CHOP antigens, were only detected in hypoxic astrocytes from $\mathrm{Tg}$ meg rats (Fig 6B). Cellular fractionation assays showed an apparent accumulation of megsin inside the ER in response to prolonged ER stress (Fig. 6C). The enhanced accumulation of megsin under ER-stress may potentially contributing to accelerated cell death of astocytes from Tg meg rats, when further stress is superimposed on this organelle. 


\section{DISCUSSION}

Megsin is a new member of the serpin superfamily cloned from a human mesangial cDNA library [6]. A role for ER stress in cellular dysfunction observed in homozygous Tg meg animals is suggested by induction of ER stress proteins in glomerular podocytes, including GRP78, GRP94 and ORP150/HSP12A [8, 9]. Presumably, the critical factor initiating widespread cellular perturbation in these animals is polymerization of megsin within the ER because of aberrant intermolecular linkage of megsin molecules (between the reactive site loop on one megsin molecule and the $\beta$-sheet of another) occurring at high local concentrations. Thus, this is a situation in which overexpression of a wild-type serpin, megsin, may result in a polymerizing species causing intracellular (ER) accumulation and cellular stress.

Familial encephalopathy with neuroserpin inclusion bodies (FENIB) typically manifests itself in the fifth decade of life and is characterized by the insidious onset of cognitive decline. Learning and memory are also affected [5]. The principal neuropathologic finding is the presence of PAS-positive inclusion bodies distributed throughout the brain, especially deeper layers of the cerebral cortex, hippocampus, and SNpc. Neurod egeneration in humans is the result of in tracellular accumulation of mutant neuroserpin, a neuron specific serpin.

In the current report, we have examined the phenotype of heterozygous $\mathrm{Tg}$ meg rats. The findings are considerably more subtle than in homozygous animals, and, thus far, are limited to the central nervous system. In homozygous Tg meg rats, PAS-positive inclusions were mainly observed in the kidney, liver, and pancreas [9]. Neurodegenerarion in homozygotes was much rapid than in heterozygotes; MAP II staining, for example, displayed almost total loss of CA1 neurons at 2 months after birth 
(not shown). Since renal and pancreatic function are also seriously impaired in homozygotes, it is difficult to assign a particular phenotype to one cause, as systemic organ failure is accompanied by a range of abnormalities (fluctuations in blood glucose, blood urea nitrogen, etc) [9].

Whereas endogenous expression of megsin in the brain is quite low/virtually undetectable, increased levels of megsin in brains of transgenic animals appears to result in progressive formation of PAS-positive inclusions, especially in deeper layers of cerebral cortex, hippocampus, and SNpc, similar to what is observed in patients with FENIB. Since animals with any renal, hepatic or pancreatic dysfunction $(\sim 20 \%$ of hetero zygotes) were excluded in this study, the abnormalities observed in our studies are most likely to have resulted from elevated expression of megsin in neurons. In this context, we propose that heterozygous Tg meg rats provide an appropriate model of FENIB.

Because of greater ease of cellular manipulation in vitro, we employed astrocytes cultured from heterozygous Tg meg rats to an alyze the cellular response to environmental perturbation. Exposure of astrocytes to hypoxia for 12 or $16 \mathrm{hrs}$ resulted in more rapid upregulation of ER stress proteins (ORP150 and GRP78), as well as expression of $\mathrm{CHOP}$ and activated caspase-12, in cultures from $\mathrm{Tg}$ meg animals versus nonTg controls. The latter findings were associated with diminished survival of astrocytes from Tg meg animals. Cellular fractionation studies demonstrated intracellular accumulation of megsin, most likely within the ER, of cultured astrocytes from Tg meg an imals exposed to hypoxia. These data are consistent with previous observations in which hypoxia slowed protein transport from the ER, thereby leading to protein accumulation $[25,26]$. Due to the relative abundance of lysine residues in 
megsin [7], this protein is a likely target of degradation via ubiquitination. Increase of intracellular accumulated megsin, possibly due to the ov erload in degradation, may requ ire further response. The ER stress response is not sufficient to overcome the challenge imposed by accumulation of megsin, and a cell death pathway involving the ER is triggered (involving activation of caspase-12) resulting in activation of the executioner capase-3. Finally, cell viability is impaired.

In conclusion, heterozygous trans genic rats overexpressing megsin provide a model of selective neuronal degeneration in which we propose that ER stress triggers a programmed cell death pathway. Though a critical question, why cellular toxicity was so selective, is under investigation, analysis of underlying mechanisms, including reasons for selective targeting of hippocampal and nigro-striatal neurons, may provide insights into pathways mediating neuronal dysfunction and death in neurodegenerative disorders associated with intracellular protein accumulation including serp inopathies. 


\section{Acknowledgements}

This study is supprted by Health and Labor Science Research Grants (H15-Kokoro-018).

We thank N. Nishihama and M. Tamatani (Department of Neuroanatomy, Kanazawa University Medical School) for expert technical support. 
References

[1] D.A. Lomas, R. Mahadeva, Alpha1-antitrypsin polymerization and the serpinopathies: pathobiology and prospects for therapy. J. Clin. Invest. 1102002 $1585-1590$

[2] R.A. Primhak, M.S. Tanner, Alpha-1 antitrypsin deficiency. Arch Dis Child. 85 $20012-5$

[3] R.W. Carrell, D.A. Lomas, Conformational disease. Lancet. 3501997 134-138

[4] R.L. Davis, A.E. Shrimpton, R.W. Carrell, D.A. Lomas, L. Gerhard, B. Baumann, D.A. Lawrence, M. Yepes, T.S. Kim, B. Ghetti, P. Piccardo, M. Takao, F. Lacbawan, M. Muenke, R.N. Sifers, C.B.Bradshaw, P.F. Kent, G.H.Collins, D. Larocca, P.D.Holohan, Association between conformational mutations in neuroserpin and onset and severity of dementia. Lancet $359 \quad 20022242-2247$

[5] R.L. Davis, P.D. Holohan, A.E. Shrimpton, A.H. Tatum, J. Daucher, G.H. Collins, R. Todd, C. Bradshaw, P. Kent, D. Feiglin, A. Rosenbaum, M.S. Yerby, C.M. Shaw, F. Lacbawan, D.A. Lawrence, Familial encephalopathy with neuroserpin inclusion bodies. Am J Pathol. 1551999 1901-1913 
[6] T. Miyata, M. Nangaku, D. Suzuki, R. Inagi, K. Uragami, H. Sakai, K. Okubo, K. Kurokawa, A mesangium-predominant gene, megsin, is a new serpin upregulated in IgA nephropathy. J Clin Invest. 1021998 828-836

[7] T. Miyata, R. Inagi, M. Nangaku, T. Imasawa, M. Sato, Y. Izuhara, D. Suzuki, A. Yoshino, H. Onogi, M. Kimura, S. Sugiyama, K. Kurokawa, Overexpression of the serpin megs in induces progressive mesangial cell proliferation and expansion. $\mathrm{J}$ Clin Invest. 1092002 585-593

[8] R. Inagi, M. Nangaku, N. Usud, A. Shimizu, H. Onogi, Y. Izuhara, K. Nakazato, Y. Ueda, H. Oishi, S. Takahashi, M. Yamamoto, D. Suzuki, K. Kurokawa, C. van Ypersele de Strihou, T. Miyata, A novel serpinopathy in rat kidney and pancreas induced by overexpression of megsin. J Am Soc Nephrol. 16 2005a 1339-1349

[9] R. Inagi, H. Onogi, M. Nangaku, H. Ueyama, Y. Kitao, S. Ogawa, K. Kurokawa, W.G. Couser, T Miyata, Involvement of endoplasmic reticulum (ER) stress in podocyte injury associated with a novel transgenic model of serpinopathy. Kidney International $682005 b$ 2639-2650

[10]K. Kuwabara, M. Matsumoto, J. Ikeda, O. Hori, S. Ogawa, Y. Maeda Y, K. Kitagawa, N. Imuta, K. Kinoshita, D. Stern, H. Yanagi, T. Kamada T, Purufucation and characterization of a novel stress protein, the $150 \mathrm{kDa}$ oxygen regulated protein (ORP150), from cultured rat astrocytes, and its expression in ischemic mouse brain. J. Biol. Chem. $27919965025-5032$ 
[11]Y. Kouroku, E. Fujita, A. Jimbo, T. Kikuchi, T. Yamagata, M.Y. Momoi, E. Kominami, K. Kuida, K. Sakamaki, S. Yonehara, T. Momoi, Polyglutamine aggregates stimulate ER stress signals and caspase-12 activation. Hum Mol Genet. $11 \quad 20021505-1515$

[12]K. Ozawa, T. Kondo, O. Hori, Y. Kitao, D. Stern, W. Eisenmenger, S. Ogawa, T. Ohshima, Expression of ORP150 (150 kDa Oxygen Regulated Protein) accelerates wound healing by modulating intracellular VEGF transport. J. Clin. Invest. 108 2001a $41-50$

[13]Y. ando, Y. Tsukamot, T. Katayama, K. Ozawa, Y. Kitao, O. Hori, D.M. Stern, A. Yamauch, S. Ogawa, ORP150/HSP12A protects renal tubular epithelium from ischemia-induced cell death. FASEB J. $18 \quad 2004$ 1401-1403

[14]X.Z. Wang, B. Lawson, J.W. Brewer, H. Zinszner, A. Sanjay, L.J. Mi, R. Boorstein, G. Kreibich, L.M. Hendershot, D. Ron, Signals from the stressed endoplasmic reticulum induce C/EBP-homologous protein (CHOP/GADD153). Mol Cell Biol. $16 \quad 19964273-4280$

[15]T. Fujikawa, S. Miguchi, N. Kanada, N. Nakai, M. Ogata, I. Suzuki, K. Nakashima, Acanthopanax senticosus Harms as a prophylactic for MPTP-induced Parkinson's disease in rats. J Ethnopharmacol. $97 \quad 2004$ 375-381 
[16]Y. Kitao, K. Ozawa, M. Miyazaki, M. Tamatani, T. Kobayashi, H. Yanagi H, M. Okabe, M. Ikawa, T. Yamashima, M. Tohyama, D. Stern, O. Hori, S. Ogawa, Expression of $150 \mathrm{kDa}$ Oxygen Regulated Protein (ORP150), a Molecular Chaperone in the Endoplasmic Reticulum, Rescues Hippocampal Neurons from Glutamate Toxicity. J. Clin. Invest. $108 \quad 2001$ 1439-1450

[17]D.Y. Zhu, S.H. Liu, H.S. Sun, Y.M. Lu, Expression of inducible nitric oxide syn thase after focal cerebral ischemia stimulates neurogenesis in the adult rodent dentate gyrus. J Neurosci. $23 \quad 2003$ 223-229

[18] S. Przedborski, V. Jackson-Lewis, R. Yokoyama, T. Shibata, V.L. Dawson, T.M. Dawson, Role of neuronal nitric oxide in 1-methyl-4-phenyl-1,2,3,6-tetrahydropyridine (MPTP)-induced dopaminergic neurotoxicity. Proc Natl Acad Sci U S A. 931996 4565-4571

[19] R.M. Wynn, J.R. Davie, P.P. Cox, D.T.Chuang, Molecular chaperones: heat-shock proteins, foldases, and matchmakers. J. Lab. Clin. Med $124 \quad 1994$ 31-36

[20] Y. Kitao, K. Hashimo to, T. Matsuyama, H. Iso, T. Tamatani, O. Hori, D.M. Stern, M. Kano, K. Ozawa, S. Ogawa, ORP150/HSP12A regulates Purkinje cell surv ival: a role for endoplasmic reticulum stress in cerebellar development. J Neurosci. 24 2004 1486- 1496 
[21] S. Ogawa, H. Gerlach, C. Esposito, A.P. Macaulay, J. Brett, D. Stern, Hypoxia modulates the barrier and coagulant function of cultured bovine endothelium. J. Clin. Invest. 851990 1090-1098

[22]K. Ozawa, Y. Tsukamoto, O. Hori, Y. Kitao, H. Yanagi, D. Stern, S. Ogawa, Regulation of tumor angiogeensis by ORP150, an inducible endoplasmic reticulum chaperone. Can. Res. 61 2001b 4206-4213

[23]B. Reaves, M. Horn, G. Banting, TGN38/41 recycles between the cell surface and the TGN: brefeldin A affects its rate of return to the TGN. Mol. Biol. Cell. 41993 93-105

[24]Hori, M. Matsumoto, M. Maeda, K. Kuwabara, H. Ueda, T. Ohtsuki, T. Kinishita, S. Ogawa, T. Kamada, D. Stern, Exposure of astrocytes to hypoxia/reoxygenation results in intracellular glucose depletion and ehnahced expression of glucose-regulated proteins (GRPs) J. Neurochem. $66 \quad 1996$ 973-979

[25] Y. Bando, S. Ogawa, A. Yamaguchi, K. Kuwabara, K. Ozawa, O. Hori, H. Yanagi, M. Tamatani, M. Tohyama, The 150 kDa Oxygen Regulated Protein (ORP150) functions as a novel molecular chaperone in the protein transport of the MDCK cells. Am. J. Physiol. (Cell Physiol.) $278 \quad 2000$ C1172-1182

[26]K. Ozawa, T, Kondo, O. Hori, Y. Kitao, D. Stern, W. Eisenmenger, S. Ogawa, T. Ohshima, Expression of ORP150 (150 kDa Oxygen Regulated Protein) accelerates 
Takano et al. / Page 23

wound healing by modulating intracellular VEGF transport. J. Clin. Invest. 108 $2001 \mathrm{a} 41-50$ 


\section{FIGURE LEGENDS}

Figure 1. Expression of megsin antigen and PAS-positive inclusions in Tg meg rats.

(A) Tissues were prepared from either $\mathrm{Tg}$ meg rats or non Tg littermates (4 months old). Protein extracts $(\sim 10 \mu \mathrm{g} / \mathrm{lane})$ were subjected to Western blotting with either anti-megsin antibody (upper panel) or anti-ORP150 antibody (middle panel), along with internal controls using anti- $\beta$-actin antibody (lower panel). (B-F) Tg meg rats (4 months old) were perfusion fixed, and brain sections was subjected to PAS staining. A representative section from the indicated brain region is shown. Dots in panel F represent three PAS-positive inclusions in a high power field (200X, approximately 1 $\mathrm{mm}^{2}$ ). A summary of microscopic observations from five different $\mathrm{Tg}$ meg rats is shown. (D-K) CA1 region brain slices prepared from either Tg meg rats (G-J) or nonTg littermates $(\mathrm{K})$ were studied by PAS staining $(\mathrm{G} \& \mathrm{~K})$. The same section $(\mathrm{G})$ was double-stained with antibodies to ORP150 (H; red) and megsin (I; green). The overlapped image is shown in panel J. Arrowheads in panels G, I, \& J indicate neuronal cells with inclusion. Data shown are representative of five repeat experiments. Abbreviations used in panels B-E, G, \& K: CA1; hippocampus CA1, $\mathrm{CCx}$; cerebral cortex, Mid; mid brain substantia nigra, $\mathrm{CbCx}$; cerebellar cortex. Scale bar, $50 \mu \mathrm{m}$.

Figure 2. Neuronal cell dea th in hippocampi of Tg meg rats. (A-F) Nissl staining was performed in rats of the indicated genotype at 4-12 months after birth. Representative micro graphs of hippocampal areas (-3.30 $\mathrm{mm}$ from the Bregma) are shown (A-F); low power (A-C; D provides hippocampus of non Tg rat at 12 months after birth) and higher power image of either CA1 of $\operatorname{Tg}$ meg (E) or Non $\operatorname{Tg}(\mathrm{F})$ at 6 
months after birth are shown. Marker bar, $50 \mu \mathrm{m}$. Quantitative analysis of undamaged neuronal density (judged by morphological criteria in Nissl staining) from CA1 is shown in panel J. Mean $\pm \mathrm{SD}$ is shown $(\mathrm{n}=8)$. ** denotes $\mathrm{p}<0.01$ by Scheffe's F test following ANOVA compared with nonTg control rats.

Figure 3. Activation of an ER stress-mediated programmed cell death pathway in the hippocampus of Tg meg rats.

(A \& B) Brain tissue was removed from rats 1-12 months after birth, and protein extracts prepared from either hippocampus (A) or cerebellar cortex (B) were subjected to Western blotting using anti-ORP150, anti-GRP78, anti-activ ated capsase-3, anti-activated caspase- 12 , or anti- $\beta$-actin antibodies. (C-I) Activated caspase-12-positive cells in the population of Neu-N-positive neurons were measured in CA1 of the animals 2-6 months after birth. Representative sections from 4 months after birth are shown in panels D-E. Sections were subjected to Nissl staining (D). Adjacent sections were immunostained with antibodies to ORP150 (E) and activated caspase-3 (F). Other sections were double stained with anti-Neu-N (G) and anti-activated caspase-12 antibodies (H), and merged images are shown in panels I. Orientation of sections (E-I, area of hippocampus) is indicated as an open box in panels D. Marker bar, $50 \mu \mathrm{m}$. Mean $\pm \mathrm{SD}$ is shown $(\mathrm{n}=8)$. ** denotes $\mathrm{p}<0.01$ by Scheffe's F test following ANOVA compared with nonTg control rats. N.D. denotes not determined due to insufficient signals (statistical analys is was not performed at this point). 
Figure 4. Neuronal loss in the SNpc of Tg meg rats. (A-E) Midbrain slic es were prepared from either Tg meg (A, C-E,) or nonTg littermates (B) 4 months after birth. Sections were subjected to PAS staining (A \& B). The same section as in A was double-stained with antibodies to megs in ( $\mathrm{C}$; green) and tyrosine hydroxylase $(\mathrm{TH} ; \mathrm{D}$, red), and merged images are shown in panel E. Marker Bar; $80 \mu \mathrm{m}$. Data shown are representative of five repeat experiments. (F-M) Coronal sections at the indicated depths from the Bregma were immunostained with anti-TH antibody (counterstained by cresyl violet) in Tg meg rats at 2 (F-I) and $6(\mathrm{~J}-\mathrm{M})$ months after birth. Marker bar, 50 $\mu \mathrm{m}$. Data shown are representative of five repeat experiments. (N \& O) Motor coordinate ability was determined by the pole test as described in text, and mean latency time to turning downward $(\mathrm{N})$ and reaching the floor $(\mathrm{O})$ is shown. ANOVA for repeated measure revealed significant differences between $\mathrm{Tg}$ meg and nonTg littermates at each time point $(\mathrm{p}<0.01)$.

Figure 5. Activation of an ER stress-mediated programmed cell death pathway in the SNpc of Tg meg rats (A-H) Midbrain sections (-5.0 $\mathrm{mm}$ from the Bregma) from either Tg meg rats (A-D) or nonTg littermates (E-H) at 4 months after birth were subjected to Nissl staining (A \&E). Adjacent sections were stained with anti-ORP150 antibody. Open boxes in panel A \& E are magnified in panels B \& F, respectively. Adjacent sections were also double-stain ed with antibodies to $\mathrm{TH}$ (green) and activated caspase-12 ( $\alpha$ casp-12; red). Merged and magnified images in the open box in panels $\mathrm{C} \& \mathrm{G}$ are shown in panels $\mathrm{D} \& \mathrm{H}$, respectively. Marker bar, $50 \mu \mathrm{m}$. Data shown are representative of five repeat experiments. (I) The same analysis was extended to animals 2 and 12 months after birth. Animals were sacrificed and sections of SNpc 
were an alyzed at four different levels $(-4.8,-5.2,-5.6$, and $-6.0 \mathrm{~mm}$ from the Bregma), and the percentage of cells staining positively for activated caspase- 12 cells in the total population of TH-positive neurons was determined in the SNpc. Mean $\pm \mathrm{SD}$ is shown $(\mathrm{n}=6) . \quad * *$ denotes $\mathrm{p}<0.01$ by Scheffe's $\mathrm{F}$ test followed by ANOVA compared with 2 mon ths old Tg meg rats. N.D. denotes not determined due to insufficient signals (statistical analysis was not performed at this point).

Figure 6. ER stress and accumulation of megsin in cultured astrocytes from Tg meg rats. Astrocyte cultures, prepared from rats of the indicated genotype were exposed to hypoxia. Cell viability at the indicated time point was assessed by dye exclusion in astrocytes. Mean $\pm \mathrm{SD}$ is shown $(\mathrm{n}=8) . \quad * *$ denotes $\mathrm{p}<0.01$ by Scheffe's $\mathrm{F}$ test following ANOVA compared with astrocytes from nonTg control rats. (B) Levels of ER stress proteins in astrocytes were determined by immunoblotting, as described in text. (C) Astrocytes $\left(\sim 5 \times 10^{6}\right.$ cells $)$ were either exposed to hypoxia or maintained in normoxia for 24 hours, and homogenates were subjected to subcellular fractionation using OptiPrep. Fractions were then subjected to dot blot analysis using anti-megsin antibody. Samples were also used for dot blot analys is with anti-mouse calnexin antibody and anti-mouse TGN38 antibody, as markers for endoplasmic reticulum and Golgi apparatus, respectively. 


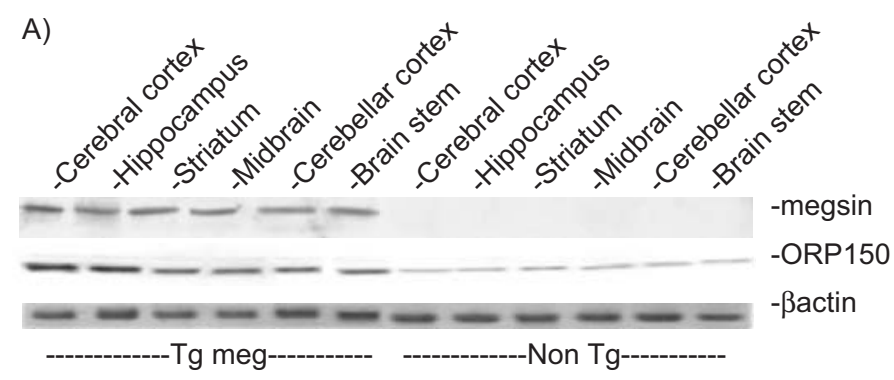

F)

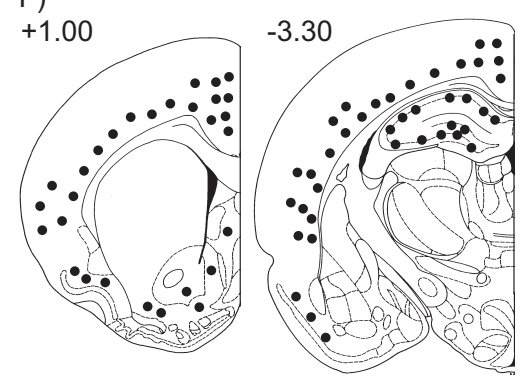

G) Tg Meg/CA1

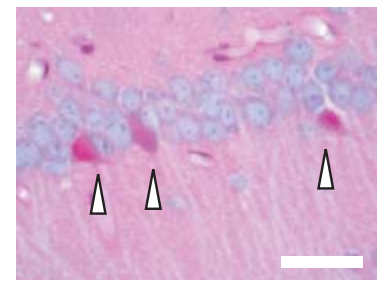

H) ORP150

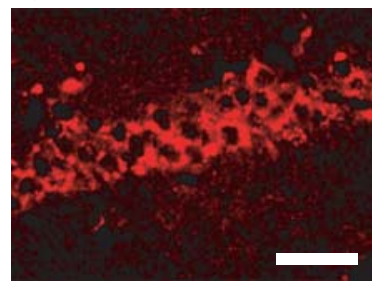

B) $\mathrm{Tg} \mathrm{Meg} / \mathrm{CA} 1$

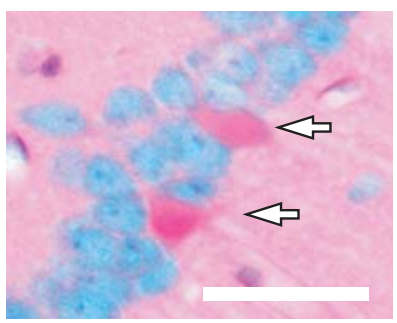

D) Tg Meg/Mid

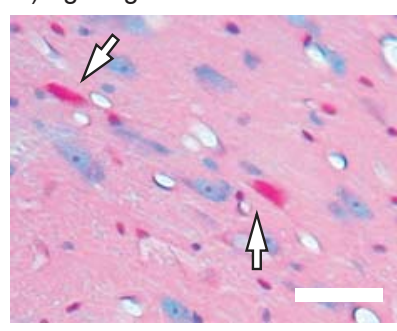

J) Merge

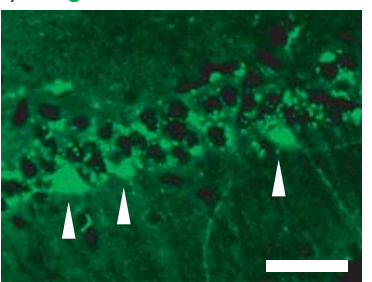

C) Tg Meg/CCx

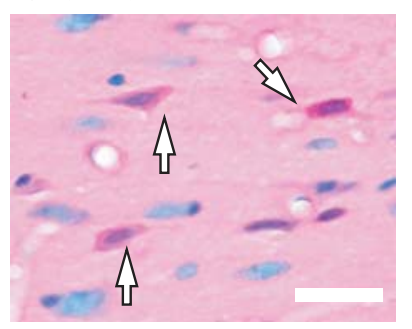

E) Tg Meg/CbCx

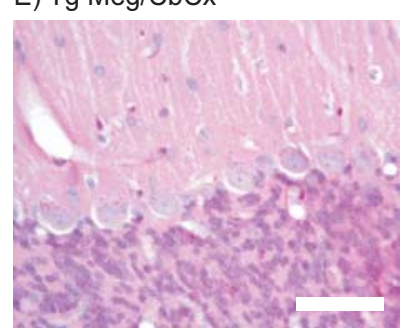

K) Non Tg/CA1

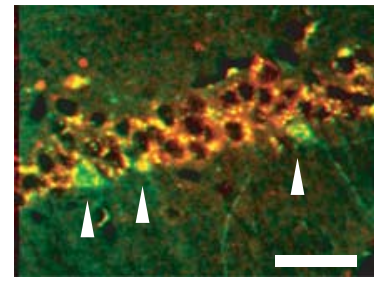

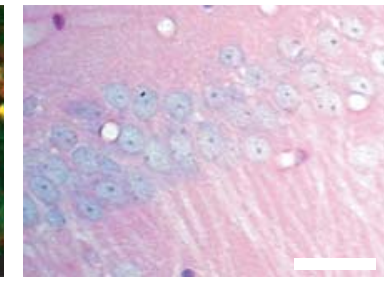

Figure 1./ Takano et al. 
Figure 2

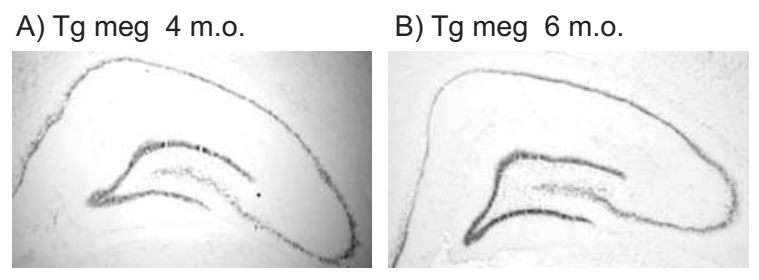

C) Tg meg 12 m.o.
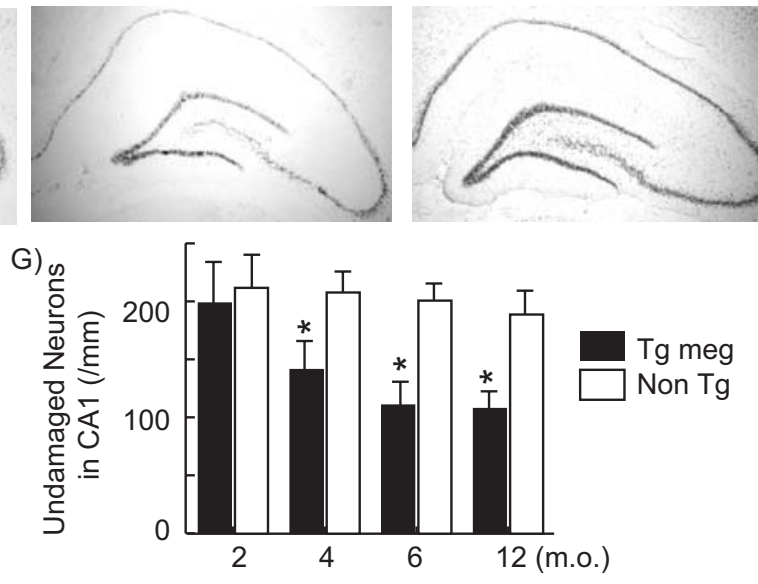

D) Non $\mathrm{Tg} 12$ m.o.

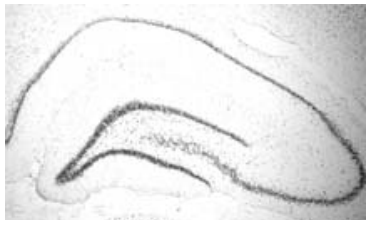

E) Tg meg / CA1 (6.m.o.) F) Non Tg / CA1 (6.m.o.)
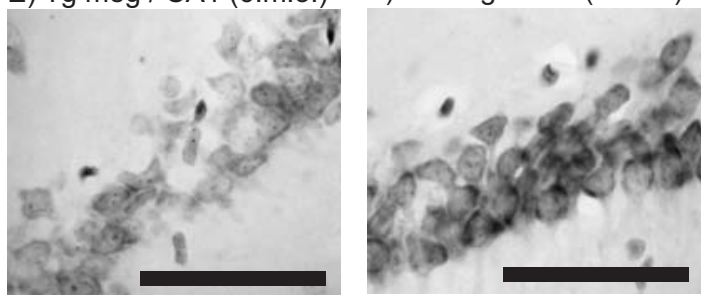

Figure 2./ Takano et al. 
Figure 3

\section{A) Hippocampus}

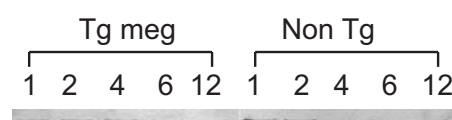

- - - - - . - - -

$-1-$
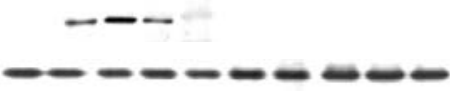

D) Nissl

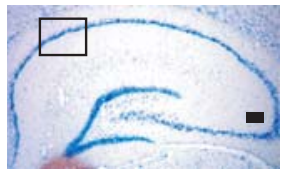

B) Cerebellar Cortex

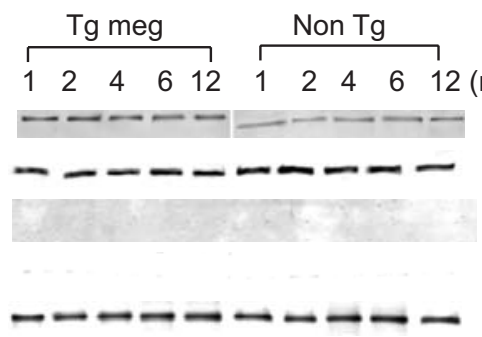

F) $\alpha$ casp-3

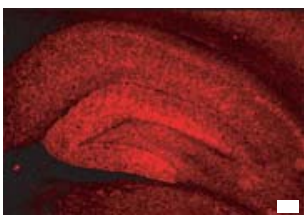

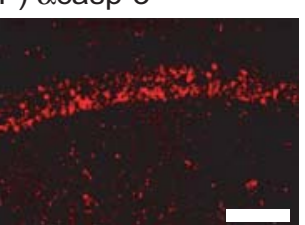

C)

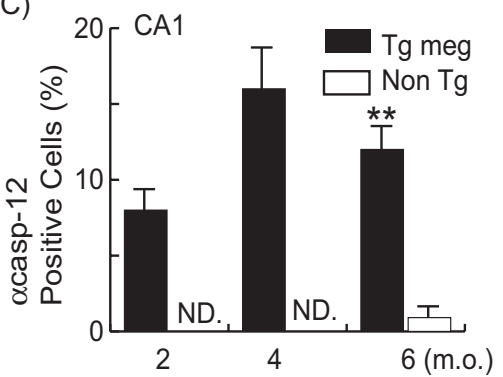

G) Neuro-N

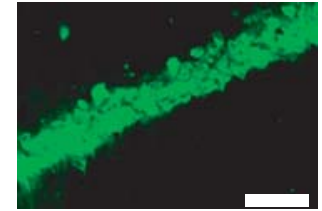

H) $\alpha$ casp-12

I) Merge

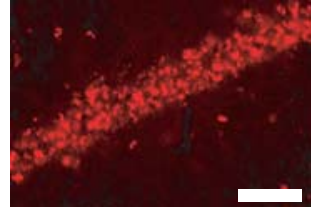

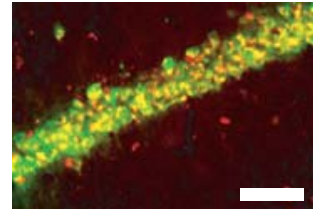

Figure 3. / Takano et al. 


\section{A) Tg meg/SNpc/PAS}

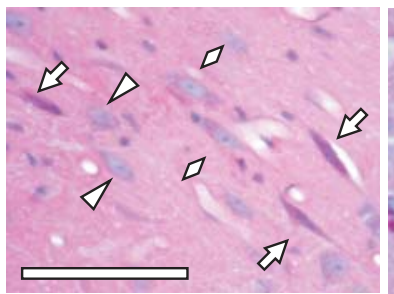

F) $-4.8 \mathrm{~mm}$
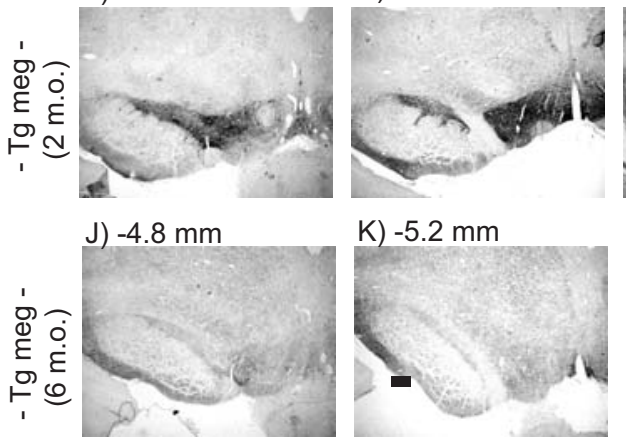

B) non $\mathrm{Tg} / \mathrm{SNpc} / \mathrm{PAS}$

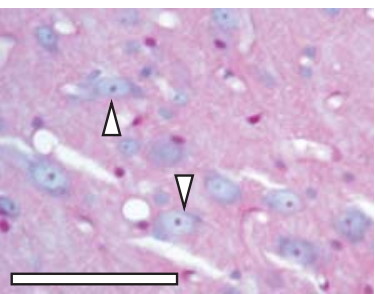

G) $-5.2 \mathrm{~mm}$

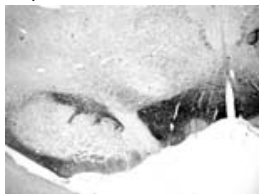

H) $-5.6 \mathrm{~mm}$

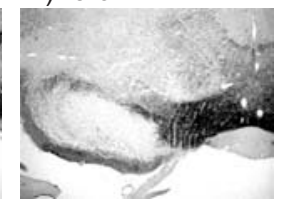

L) $-5.6 \mathrm{~mm}$
C) Tg meg/SNpc/Megsin

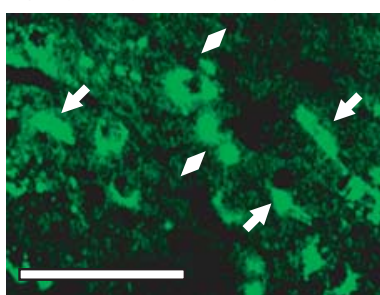

I) $-6.0 \mathrm{~mm}$

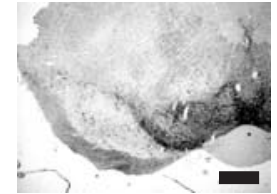

M) $-6.0 \mathrm{~mm}$
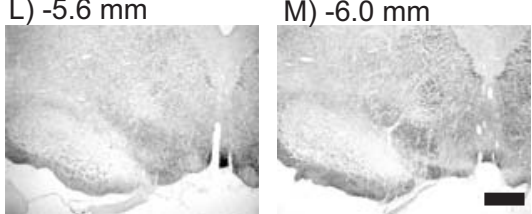

D) $\mathrm{Tg}$ meg/SNpc/TH

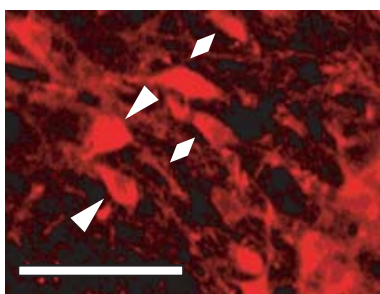

N)

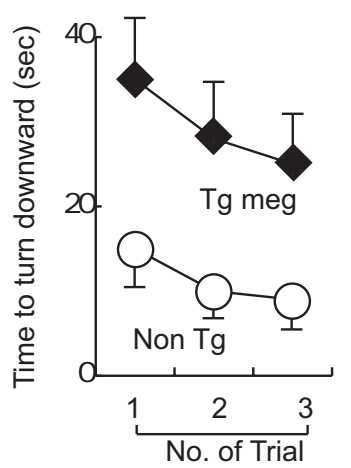

E) Tg meg/SNpc/Merge

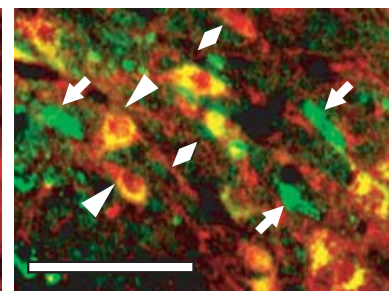

O)

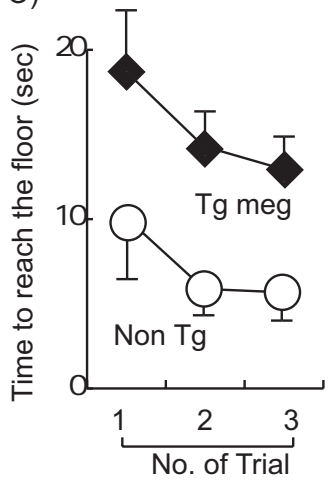

Figure 4. / Takano et al. 
Figure 5
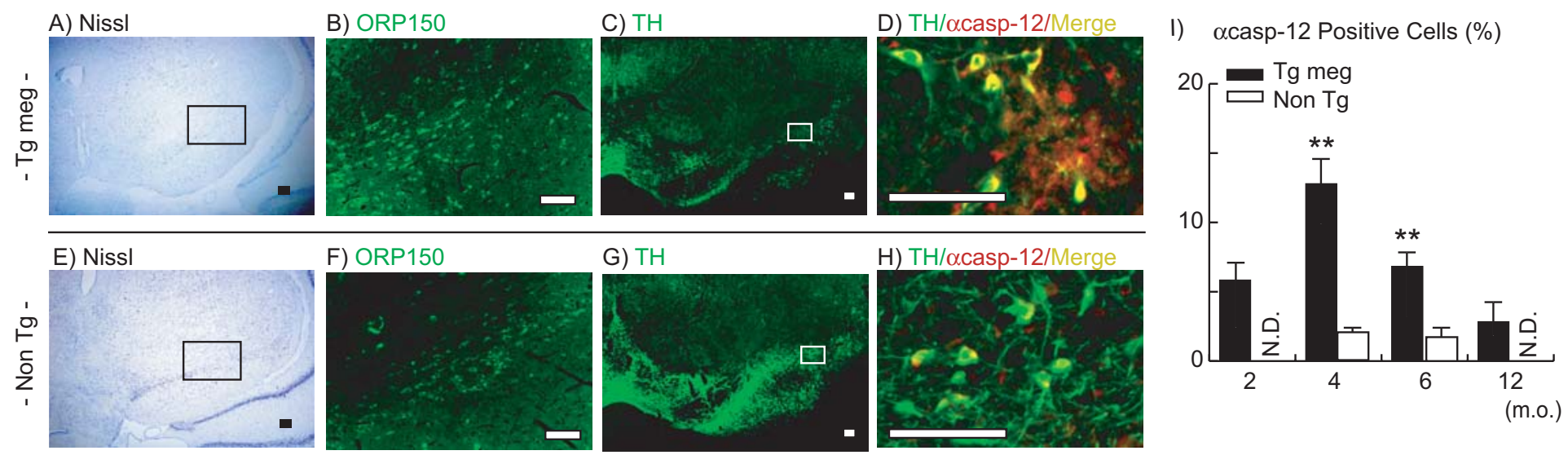

Figure 5. / Takano et al. 
Figure 6

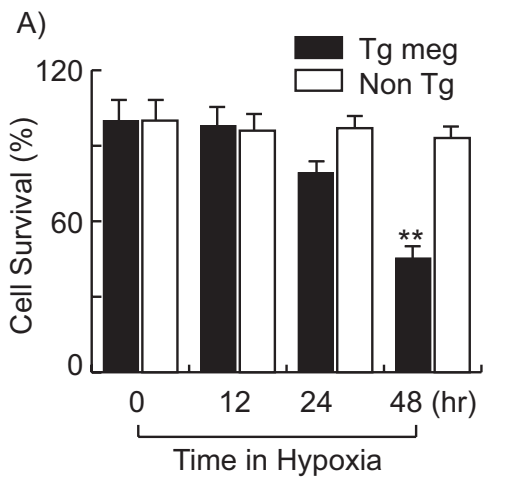

B)

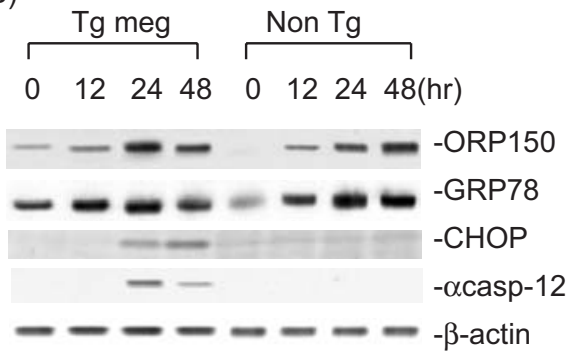

C) \begin{tabular}{llllllllll}
\hline 1 & 2 & 3 & 4 & 5 & 6 & 7 & 8 & 9 & 10
\end{tabular}

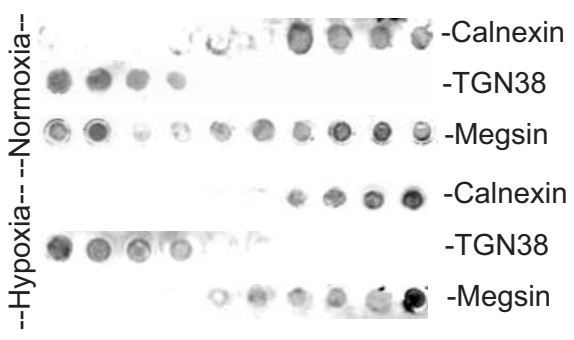

Figure 6./ Takano et al. 